\title{
Arrancador Progresivo para Motores de Inducción Trifásicos Mediante Programación Arduino
}

\section{Progressive Starter for Three Phase Induction Motors Through Arduino Programming}

\author{
CABRERA-ORNELAS, Javier†*, VELAZQUEZ-ROMERO, Miguel Ángel, BRITO-MONROY, Jorge \\ y HERNÁNDEZ-NARVÁEZ, Ana Luisa
}

Tecnológico de Estudios Superiores de Jilotepec

ID $1^{\mathrm{er}}$ Autor: Javier, Cabrera-Ornelas / ORC ID: 0000-0002-9398-1498, CVU CONACYT ID: IT18B386

ID $1^{\mathrm{er}}$ Coautor: Miguel Ángel, Velazquez-Romero / ORC ID: 0000-0003-3087-289X

ID $2^{\text {do }}$ Coautor: Jorge, Brito-Monroy / ORC ID: 0000-0002-3273-4872

ID $3^{\text {do }}$ Coautor: Ana Luisa, Hernández-Narváez / ORC ID: 0000-0003-4500-7209, CVU CONACYT ID: IT19A966

DOI: $10.35429 /$ JTIP.2019.8.3.15.20

Recibido 16 de Julio, 2019; Aceptado 19 Septiembre, 2019

\begin{abstract}
Resumen
El arranque en forma progresiva de los motores industriales de inducción de corriente alterna (CA) es indispensable en cualquier proceso industrial, en la actualidad se cuenta con equipo muy confiable que realiza la variación de velocidad en forma progresiva, pero estos equipos son de alto costo. En este artículo se presenta una alternativa para poner en funcionamientos motores trifásicos de inducción en una forma progresiva hasta llegar a su velocidad nominal, la cual consiste en usar una placa Arduino para la parte de control y poder variar las resistencias externas del circuito de potencia el cual nos variara al ángulo de operación de la señal alterna, el circuito de potencia consiste en emplear un circuito RC y dispositivos tiristores de potencia que a través del control nos permitan modificar las resistencia del circuito RC y así variar el ángulo de disparo del tiristor. Como se muestra en el contenido del trabajo el control es muy fácil de operar y de un costo económico, por lo que el principio se podría implementar en forma sustentable en otras aplicaciones, como son, variadores de velocidad industriales.
\end{abstract}

Variación, Motor, Inducción

\begin{abstract}
The progressive start of the industrial induction motors of alternating current $(\mathrm{AC})$ is indispensable in any industrial process, at the present time it is counted on very reliable equipment that realize the variation of speed in progressive form, but these equipments are of high cost . In this article an alternative is presented to put into operation three-phase induction motors in a progressive way until reaching its nominal speed, which consists in using an Arduino plate for the control part and to be able to vary the external resistances of the power circuit. which will vary us to the angle of operation of the alternating signal, the power circuit consists of using an RC circuit and thyristor power devices that through the control allow us to modify the resistance of the RC circuit and thus vary the firing angle of the thyristor. As shown in the content of the work control is very easy to operate and of an economic cost, so the principle could be implemented in a sustainable way in other applications, such as industrial speed variators.
\end{abstract}

Variation, Motor, Induction

Citación: CABRERA-ORNELAS, Javier, VELAZQUEZ-ROMERO, Miguel Ángel, BRITO-MONROY, Jorge y HERNÁNDEZ-NARVÁEZ, Ana Luisa. Arrancador Progresivo para Motores de Inducción Trifásicos Mediante Programación Arduino. Revista de Tecnologías en Procesos Industriales. 2019 3-8: 15-20

\footnotetext{
* Correspondencia del Autor (Correo electrónico: javier.cabrera@ hotmail.com)

$\dagger$ Investigador contribuyendo como primer autor.
} 


\section{Introducción}

Los controles programables mediante el uso de la electrónica hoy en día forman parte importante en la mayoría de los procesos de automatización y control industrial los cuales requieren una operación precisa, este tipo de controles en la actualidad se encuentran en forma activa en el campo residencia, industrial y militar.

En la actualidad existen varios equipos que realizan el arranque progresivo de los motores de inducción los cuales son aplicados en la mayoría de los procesos industriales con una gran precisión pero el inconveniente es el elevado costo de mercado y en muchas ocasiones el arranque progresivo es fijo. La alternativa que se presenta es mediante la tarjeta electrónica programable Arduino la cual es de bajo costo y no requiere de licencia para el software de programación.

El método que se presenta en este trabajo para realizar la etapa de control que nos genere las salidas digitales progresivas es mediante el uso de la tarjeta Arduino, y que a la vez las salidas digitales seleccionen los diferentes valores de las resistencias las cuales nos proporcionaran un ángulo de disparo progresivo en la etapa de potencia. La etapa de potencia consiste en el uso de dispositivos electrónicos de potencia (Tiristores).

Debido al bajo consumo de energía eléctrica que se necesita para activar estos dispositivos electrónicos, el circuito de control puede activar la mayoría de los dispositivos electrónicos de estado sólido de bajo costo como capacitores resistencias, diodos, opto acopladores y circuitos integrados.

Este tipo de control, utilizado para procesos automáticos de arranque de motores de inducción es verdaderamente practico y puede aplicarse en forma sustentable en cualquier proceso en el cual se requiera de un arranque secuencial de motores los cuales estén acoplados a un par de carga como bandas transportadoras o ventiladores y que tienen la necesidad de arrancar en forma automática o forma manual.

Con este trabajo se pretende arrancar los motores de inducción en forma progresiva para evitar el alto consumo de corriente en el arranque lo que se ve reflejado en el costo por consumo eléctrico.

\section{Desarrollo y construcción del arrancador progresivo}

En esta sección se pretende explicar la función de cada uno de los componentes empleados en el arrancador progresivo.

\section{Placa de Arduino uno}

Es un sistema de programación de los lenguajes c/c++, con plataforma electrónica que utiliza micro controladores para formar sistemas especializados en la automatización de procesos, con un amplio campo de aplicación en ciencias exactas y en ingeniería. Como es una arquitectura abierta en sus modelos y es lo que lo hace atractivo para desarrollar aplicaciones de automatización ya que es adecuado para cualquier proceso físico ya que tiene software libre.

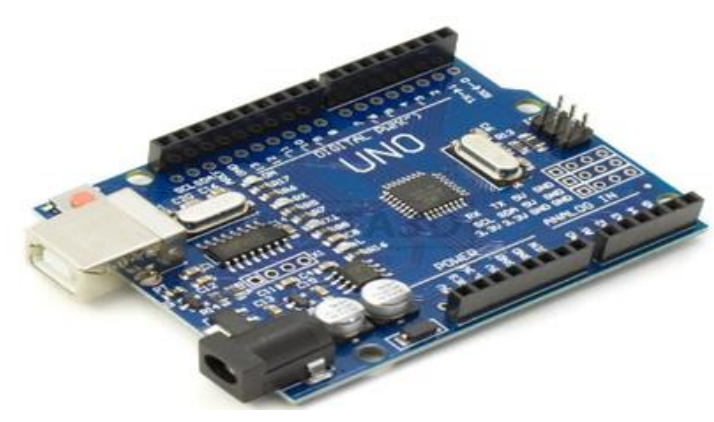

Figura 1 Placa Arduino.

Fuente: Fuente: www.electronicaymas.com,2019

\section{Triac}

El triac es un interruptor del tipo semiconductor de CA de tres terminales que se activa cuando se aplica una señal de baja energía a su puerta. Este dispositivo conduce corriente en cualquier dirección cuando es activado por la compuerta. Una señal de puerta positiva o negativa activará al dispositivo, además el triac puede ser pensado como dos SCR complementarios en paralelo.
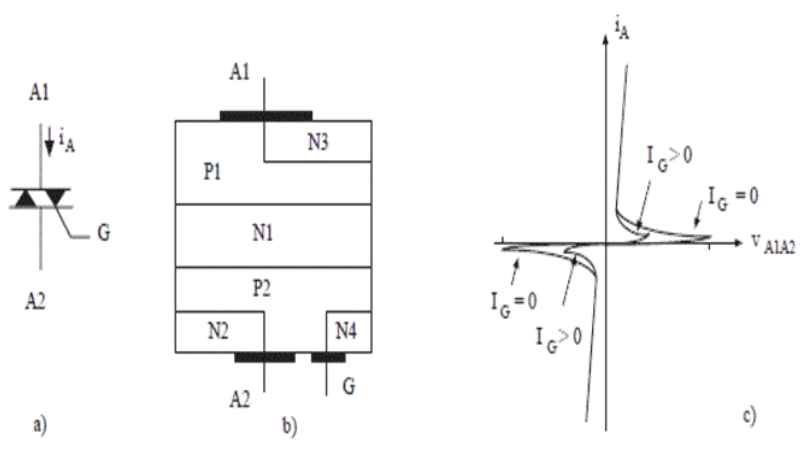

Figura 2 Símbolo, composición y características eléctricas del triac.

Fuente: Elaboración Propia

CABRERA-ORNELAS, Javier, VELAZQUEZ-ROMERO, Miguel Ángel, BRITO-MONROY, Jorge y HERNÁNDEZ-NARVÁEZ, Ana Luisa. Arrancador Progresivo para Motores de Inducción Trifásicos Mediante Programación Arduino. Revista de Tecnologías en Procesos Industriales. 2019 


\section{Sistema RC}

Un sistema RC, como el que se muestra en la Fig. 3, está constituido de los siguientes componentes:

1. El conmutador tiene tres posiciones:

- $\quad$ Posición S1 no hay carga del capacitor

- Posición S2 carga al capacitor

- $\quad$ Posición S3 descarga al capacitor

2. El circuito consta de un voltaje que carga a un capacitor

3. El capacitor está conectado en serie con una resistencia

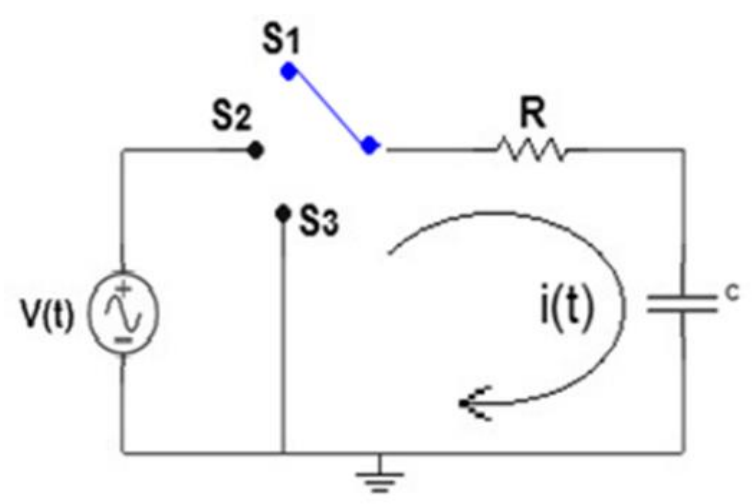

Figura 3 Circuito RC

Fuente: Elaboración Propia

Los elementos que intervienen en el circuito RC, del sistema de estudio se presentan en la tabla 1 .

\begin{tabular}{|c|c|c|}
\hline Elemento & Símbolo & $\begin{array}{l}\text { Unidades de } \\
\text { medida }\end{array}$ \\
\hline $\begin{array}{l}\text { Generador de corriente } \\
\text { alterna } \\
\text { entrada) }\end{array}$ & $\infty$ & Volts (V) \\
\hline Conmutador & $\stackrel{s_{3}}{s_{2}}$ & Off-On \\
\hline Resistencia & $-\stackrel{R}{R}$ & $\operatorname{Ohms}(\Omega)$ \\
\hline Capacitancia & $=c$ & Faradios $(\mathrm{F})$ \\
\hline Corriente & $i(t)$ & Amperes (A) \\
\hline
\end{tabular}

Tabla 1 Elementos del sistema RC

Fuente: Elaboración Propia

\section{Relevador}

Un relevador es un dispositivo electromecánico. Funciona mediante la energización de una bobina la cual produce un campo magnético que produce el cierre o apertura de contactos mecánicos los cuales son aprovechados para la activación de dispositivos de corriente alterna (CA) o corriente directa (CD).
Septiembre, 2019 Vol.3 No.8 15-20

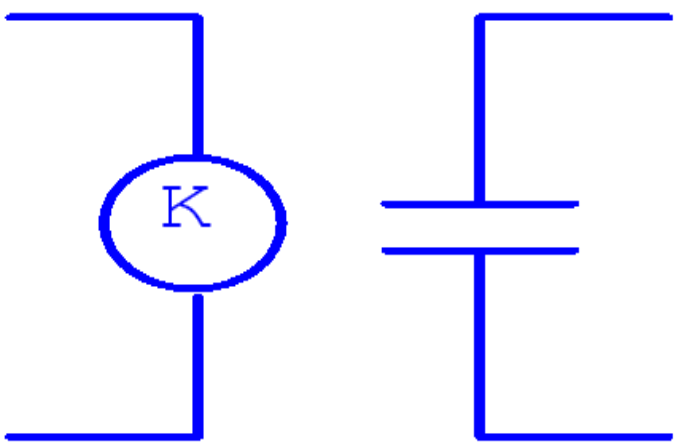

Figura 4 Relevador

Fuente: Elaboración Propia

\section{Metodología a desarrollar}

$\mathrm{El}$ arrancador progresivo se basa principalmente en dos etapas, la primer etapa es la de control la cual consiste en la programación de la placa Arduino de tal manera que proporcione por sus salidas digitales un número digital ascendente el cual se utilizara para variar la resistencia de control de la segunda etapa que es la potencia.

La etapa de potencia consiste en la variación de la resistencia de control la cual se encargara de variar progresivamente el valor energético del voltaje entregado a la carga que en este caso será un motor de inducción, la variación será en un pequeño periodo de tiempo aproximadamente de 1 a 2 segundos que es el tiempo promedio que tarda un motor de inducción en llegar a su velocidad nominal.

\section{Etapa de control}

La etapa de control consiste en programar mediante el entorno de desarrollo integrado (IDE) de Arduino el programa que sea capaz de realizar la siguiente lógica mostrada en la tabla 2.

\begin{tabular}{l|l|l|}
\hline $\begin{array}{l}\text { \#Entrada } \begin{array}{l}\text { \# Salida digital } \\
\text { digital arranque }\end{array} \\
\text { Grados eléctricos } \\
\text { activados }\end{array}$ \\
\hline 0 & 1 & 90 \\
\hline & 2 & 80 \\
\hline & 3 & 70 \\
\hline & 4 & 60 \\
\hline & 5 & 50 \\
\hline & 6 & 40 \\
\hline & 7 & 30 \\
\hline & 8 & 20 \\
\hline & 9 & 10 \\
\hline & 10 & 0 \\
\hline
\end{tabular}

Tabla 2 Tabla de verdad del arrancador progresivo Fuente: Elaboración Propia 
La finalidad de la programación es que mediante un pulso de arranque por la entrada digital 0 de Arduino se active la lógica de salida la cual nos proporcione una salida digital en alto cada segundo según las salidas mostradas en la tabla 2.

\section{Etapa de potencia}

La etapa de potencia consiste en el uso de tiristores, en este caso en particular se empleó un triac ya que en la actualidad tienen una gran aplicación industrial en las etapas de potencia y son capaces de conmutar en ambos semiciclos de la corriente alterna.

\section{Control de fase (metodología)}

Un método eficaz y ampliamente utilizado de control de la potencia media a una carga es a través del triac y es por control de fase. Control de fase es un método mediante el cual se utiliza el triac para aplicar el suministro de corriente alterna a la carga por una fracción controlada de cada ciclo. El gráfico 1 muestra la onda de tensión junto con los ángulos de disparo y conducción que se utilizan para el funcionamiento del triac.

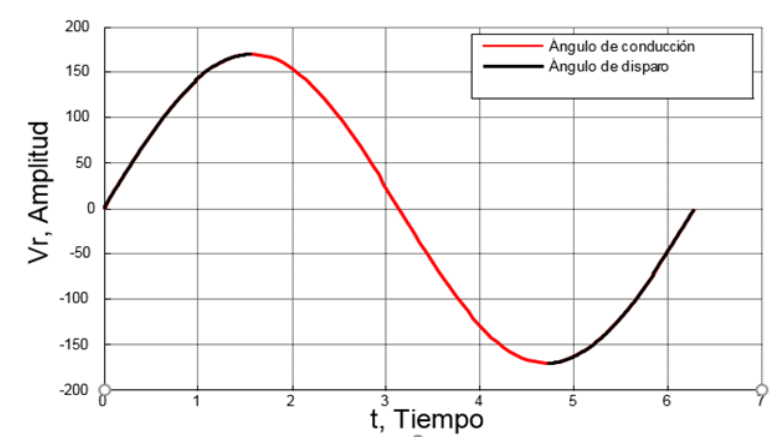

Gráfico 1 Control de fase

\section{Circuito de control de compuerta triac}

Para el sistema circuito RC que se muestra en la figura 3 , se conecta un circuito de control de compuerta para un triac, como se ilustra en la figura 6

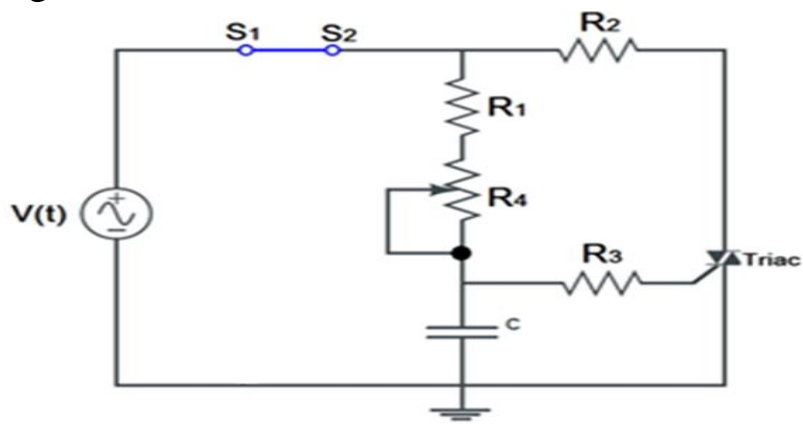

Figura 6 Sistema RC para el control del triac
La función del triac por sus características bidireccionales, en su estado de conducción queda controlado por el potencial de disparo aplicado a la compuerta y la dirección de la corriente eléctrica queda determinada por la polaridad del potencial que recibe (Zetina, 2000).

Una corriente (IG) se aplica a la compuerta del triac, la cual fluye a través de la unión entre la compuerta y la terminal MT1, la cantidad de corriente de compuerta para disparar un triac es una corriente establecida IGT. Una vez que el triac se ha disparado, mientras que la corriente continúe su flujo a través de las terminales principales, de MT2 a MT1, el triac permanecerá encendido (San Miguel, 2014).

Donde, $\mathrm{R}_{4}$ es una resistencia variable que tomará el valor ideal para disparar el triac de acuerdo a las salidas digitales de la etapa de control para una señal senoidal.

\section{Resultados: Sistema de disparo del triac mediante resistencias.}

Para la implementación del método propuesto se usó un circuito como el que se muestra en la figura 6 , para accionar una carga en un tiempo establecido.

La tabla 3 describe las características de los elementos del circuito de estudio.

\begin{tabular}{|l|l|}
\hline \multicolumn{2}{|c|}{ Parámetro } \\
\hline R3 & $100.0 \Omega$ \\
\hline R4 & 1 a $500.0 \Omega$ \\
\hline C & $0.01 \mu \mathrm{F}$ \\
\hline $\mathrm{V}(\mathrm{t})$ & $120 \mathrm{Vrms}, 60 \mathrm{~Hz} .0^{\circ}$ \\
\hline & $\begin{array}{l}\text { 2N6073 } \\
\text { VGT: } 2.5 \text { máx. } \\
\text { Triac }\end{array}$ \\
& I GT: 30mA. \\
\hline
\end{tabular}

Tabla 3 Características del circuito de estudio Fuente: Elaboración Propia

El objetivo consiste en calcular el valor de la resistencia variable para disparar el triac en un tiempo determinado utilizando la ecuación 1.

$i(t)=\frac{A C w}{C^{2} R^{2} w^{2}+1}\left[-e^{\frac{t}{C R}}+\cos (w t)+C R w \sin (w \mathrm{t})\right] A m p$

Siendo:

$\left(R_{1}+R_{4}\right) \mathrm{C}$

CABRERA-ORNELAS, Javier, VELAZQUEZ-ROMERO, Miguel Ángel, BRITO-MONROY, Jorge y HERNÁNDEZ-NARVÁEZ, Ana Luisa. Arrancador Progresivo para Motores de Inducción Trifásicos Mediante Programación Arduino. Revista de Tecnologías en Procesos Industriales. 2019 
La constante de tiempo mínima ocurre cuando $R_{4}$ está ajustada hacia un extremo, siendo:

$R_{1}=200 k \Omega$

La constante de tiempo máxima ocurre cuando $R_{1}$ está completamente hacia el otro extremo, es decir:

$R_{4}=2320 k \Omega$

Considerando valores estándar comerciales mostrados en la tabla 4.

\begin{tabular}{|l|l|}
\hline Parámetro & \multicolumn{1}{|c|}{ Valor } \\
\hline R1 & $1.0 \mathrm{k} \Omega$ \\
\hline \multirow{4}{*}{ R4 } & $1.0 \mathrm{k} \Omega$ \\
\cline { 2 - 2 } & $3.3 \mathrm{k} \Omega$ \\
\cline { 2 - 2 } & $3.9 \mathrm{k} \Omega$ \\
\cline { 2 - 2 } & $4.7 \mathrm{k} \Omega$ \\
\hline
\end{tabular}

Tabla 4 Valores de R1 y R4

Fuente: Elaboración Propia

Sustituyendo los valores en la ecuación 5 de la resistencia, dado que el voltaje alcanza 2.5 volts en un ángulo eléctrico de $90^{\circ}$, se disparara el triac, como se muestra en el gráfico 2.

$$
V_{R}=R i(t)=\frac{A C R w}{C^{2} R^{2} w^{2}+1}\left[-e^{\frac{-t}{C R}}+\cos (w t)+C R w \operatorname{sen}(w t)\right]
$$

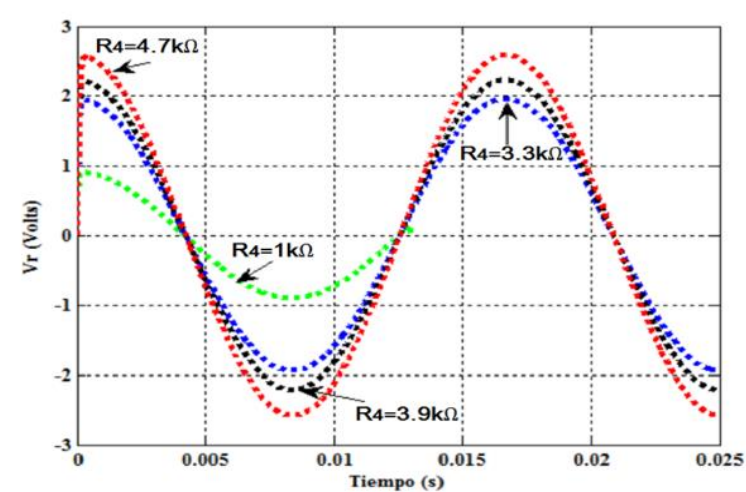

Gráfico 2 Vr para diferentes valores de R4 Fuente: Elaboración Propia

Se logra variar el ángulo de 0 a $90^{\circ}$ eléctricos y con esto variar el valor energético a la carga y a la vez que el motor de inducción tenga un arranque más suave y consuma menos corriente.

\section{Anexos}

Los gráficos que se muestran son las obtenidas en el laboratorio sobre las terminales de un motor de inducción, las características generales se muestran en la siguiente tabla.

\begin{tabular}{|l|l|}
\hline \multicolumn{2}{|c|}{ Valores de placa } \\
\hline$V_{a}=120 \quad$ Volt & $B=0.006 \mathrm{~N} . \mathrm{m}$ \\
\hline$I_{a}=2.5 \quad \mathrm{Amp}$ & $K=0.57$ \\
\hline$R P M=1800$ & $R_{a}=5 \quad \Omega$ \\
\hline$T_{r}=1.14$ & $L_{a}=0.043 \mathrm{H}$ \\
\hline$H P=1 / 4$ & $J=0.57 \mathrm{Kg} . \mathrm{m}^{2}$ \\
\hline
\end{tabular}

Tabla 5 Características nominales del motor de inducción Fuente: Elaboración Propia
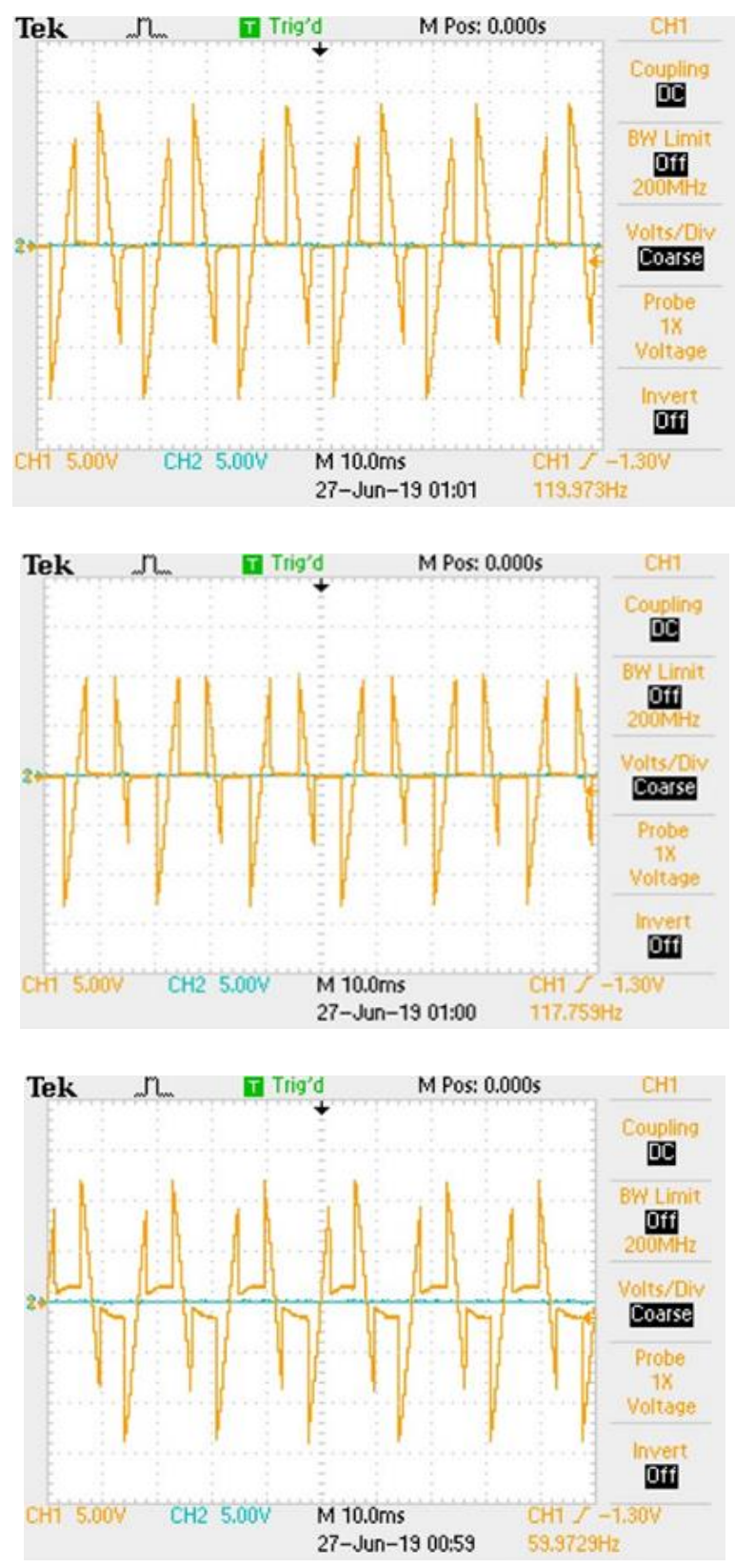

Gráfico 3 Variación del ángulo de disparo Fuente: Elaboración Propia

\section{Agradecimiento}

El primer autor agradece al Tecnológico de Estudios Superiores de Jilotepec por el apoyo institucional y de sus laboratorios para las pruebas necesarias para la realización de este trabajo de desarrollo tecnológico.

CABRERA-ORNELAS, Javier, VELAZQUEZ-ROMERO, Miguel Ángel, BRITO-MONROY, Jorge y HERNÁNDEZ-NARVÁEZ, Ana Luisa. Arrancador Progresivo para Motores de Inducción Trifásicos Mediante Programación Arduino. Revista de Tecnologías en Procesos Industriales. 2019 


\section{Conclusiones}

Existen diferentes aplicaciones para el control de la velocidad de un motor de inducción, en la industria surgen diariamente los retos por gestionar y optimizar costos. En los tiempos llamados set off, y los horarios de menor demanda dentro de las empresas se deben generar las menores perdidas en cuanto a costo. Este proyecto está planteado de manera práctica para conocer, ubicar y seleccionar el nivel de voltaje que fácilmente podría cambiarse dentro de la dinámica original, también puede considerarse para la implementación de un controlador proporcional derivativo (PD) que permita cambiar automáticamente la velocidad del motor como el proceso lo requiera.

Las ventajas de emplear variadores estáticos están en el abaratamiento de costos de los componentes electrónicos de potencia. También se sabe que la forma de onda de la tensión aplicada al motor no es senoidal.

El inconveniente que se pueda suponer a esta distorsión de la forma de onda, y por tanto las perdidas adicionales a las que da lugar, se compensan sobradamente con la simplicidad del procedimiento y, sobre todo, por lo eliminación del transitorio de arranque. Hay que tener en cuenta que para potencias pequeñas (de unos pocos $\mathrm{kW}$ ), el coste del arrancador es varias veces superior al de propio motor. El estudio realizado se tiene pensado escalonarlo a un motor trifásico.

\section{Referencias}

Albert Malvino y David J. Bates. Principios de electrónica Editorial McGraw-Hill, España, 2007

Antonio Hermosa Donate. Electronica aplicada Editorial Alfaomega, México 2013.

\begin{tabular}{|c|c|c|}
\hline $\begin{array}{l}\text { Boylestad Robert } \\
\text { Louis. Electrónica. }\end{array}$ & & $\begin{array}{r}\text { Nashelsky } \\
\text { Teoría }\end{array}$ \\
\hline de Circuitos. Editorial & Prentice & Hall \\
\hline
\end{tabular}

Días, A., Estéfani, D., Morocho. G., \& Cecilia. G. (2019). Estudio comparativo entre el sistema de bombeo electrosumergible con motor de inducción y el sistema de bombeo electrosumergible con motor de iman permanente en el campo Sacha.
Duarte-Carvajalino, J. M., Guerrero-Díaz, O., \& Carbajal-Labastida. C (2019). Estimación de los parámetros de motores de inducción a partir de las medidas de pérdidas de potencia. Revista UIS Ingenierías, 175-182.

Guanochanga, C., Andrés, C., \& Tenorio Chango, C. M. (2019). Diseño e implementación de un sistema de monitoreo, para el análisis dinámico de un motor eléctrico asíncrono trifásico de $3 \mathrm{HP}$, en el laboratorio de control Eléctrico de la Universidad de las Fuerzas Armadas ESPE Extensión Latacunga (Bachelor's thesis, Universidad de las Fuerzas Armadas ESPE Extensión Latacumba. Carrera de Ingeniería Electromecánica.

http://www.datasheetcatalog.net/es/datasheets_ $\mathrm{pdf} / \mathrm{M} / \mathrm{O} / \mathrm{C} / 3 / \mathrm{MOC} 3010 . \mathrm{shtml}$

http://www.onsemi.com/pub_link/Collateral/BT A08-600BW3-D.PDF

Luis, A., \& Beatriz, S. (2019). Diiseño de sistemas de control de velocidad de un motor asíncrono trifásico con Arduino.

Maloney J. Timothy. Electrónica Industrial. Dispositivos y Sistemas. Editorial Prentice Hall Hispanoamericana, México, 2000

Manual de reemplazos ECG. Silvania, USA, 2000

Morales, J. Y. R. Beltran, A. M., Azcaray, H. R., Santiago, K., Calixto, -m., \& Sarmiento, E. (2019). Simulación y control de la velocidad y par electromagnético de un motor de inducción trifásico: Un enfoque a vehículos eléctricos. Revista Iberoamericana de Automática e Informática Industrial, 16(3), 308-320.

Reyes Cortes, Fernando; Cid Monjaraz, Jaime; Arduino. Aplicaciones en Robótica, Mecatrónica e Ingenierías; Alfaomega.

Williams, Arthur. Microprocesadores, dispositivos optoelectrónicos, periféricos y de interfaz. Serie de circuitos integrados. Mc Graw Hill, México, 1989. 